\section{Tratamiento quirúrgico y complicaciones de lesiones esofágicas; estudio de casos en Hospital Escuela, Honduras}

Surgical Management and Complications of esofhageal injuries; Study of cases in Hospital Escuela, Honduras

C.E.R ${ }^{1}$

Dra. Martha Ayes Rivera ${ }^{2}$

Dr. Francisco Ayes Valladares ${ }^{3}$

1 Comité Editorial de la Revista de Postgrados de Medicina UNAH, Dirección de Docencia e Investigación-HE: Dr. Carlos Vargas Pineda, Dr. Rolando-Aguilera-L, Dra. Guadalupe-Romero-A

2 Médico Residente III año Cirugía General, UNAH.

3 Cirujano Torácico General, Profesor Titular III, UNAH.

RESUMEN. OBJETIVO. Establecer la prevalencia, el manejo quirúrgico, así como la morbilidad y mortalidad asociadas a lesiones esofágicas.

PACIENTES Y MÉTODOS. Estudio descriptivo transversal que involucró a los pacientes que ingresaron al Servicio de Urgencias Quirúrgicas del Hospital Escuela entre el $1^{\circ}$ de enero de 2008 y el 30 de junio de 2010 con diagnóstico de lesión esofágica. Se aplicó un Instrumento de recolección de datos clínicos y de manejo del paciente. Los resultados se presentan como frecuencias y porcentajes de las variables estudiadas utilizando el sistema de proceso de datos EPI.INFO 3.5.1

RESULTADOS. Se incluyeron en el estudio 11 casos de pacientes con lesiones esofágicas que se manejaron en el servicio de urgencias quirúrgicas del Hospital Escuela, la mayoría de los casos se dio en el sexo masculino, con una edad promedio de 29 años. La principal etiología de estas lesiones fue la herida por arma de fuego en el $64 \%$ de los casos y secundaria a cuerpos extraños (placa dental) en el $27 \%$. La zona anatómica más afectada fue la porción cervical en un $64 \%$ seguida de la torácica; el grado de lesión esofágica más frecuente fue la de grado III manejada en la mayoría de los casos mediante cierre primario. La exclusión esofágica se realizó en el $36.4 \%$ de los casos y esto a consecuencia del daño tisular y el grado de contaminación encontrado al momento del procedimiento quirúrgico.
Los estudios de imagen realizados para la detección de estas lesiones fueron la radiografía cervical y torácica y el esófagograma, en ninguno de los casos se realizó endoscopia.

La principal complicación de estas lesiones fue la mediastinitis que llevó a la muerte a 4 de los pacientes de esta serie.

CONCLUSIón. Al igual que en otros países las lesiones esofágicas son raras, su sintomatología vaga hace que en ocasiones pasen desapercibidas, sin embargo la alta letalidad de sus complicaciones hace necesario su manejo oportuno.

ABSTRACT.OBJECTIVE. Establish the prevalence and surgical treatment of esophageal injuries and the morbidity and mortality associated.

PATIENTS AND METHODS. Descriptive study involving patients admitted to the surgery service of Hospital Escuela from January $1^{\text {st, }}$ 2008 through june $30^{\text {th }}, 2010$ with diagnosis of esophageal injuries. We applied a data collection instrument for clinical findings and patient management. The results are presented as frequencies and percentages of study variables using the data processing system EPI.INFO 3.5.1

RESULTS. A descriptive clinical review was made of all patients with perforation of tha esophagus. There were 11 patients the most of them males an average age of 29 years. The principal cause of injury was gun shut $64 \%$ of cases follow by foreign body ( $27 \%$ ). Cervical esophagus was the most affected area 64\% follow by the intrathoracic esophagus. Grade III injuries were the most frequent and it was manage with suture of the perforation. Esophageal exclusion was performed in the $36.5 \%$ of the cases this due to the severity of tissue damage found. Image studies performed were cervical $X$ ray, thoracic projections and esophagography. The most serious complication of this injuries was the mediatinitis with a high mortality in 4 of the 11 cases..

CONCLUSION. Esophageal injuries are rare conditions with a high mortality that makes important to diagnose and treat this injuries as soon as they are found.

Keywords: esophageal injuries, mediastinitis 
INTRODUCCION.

La lesión del esófago, aunque relativamente rara, representa un dilema para los cirujanos, especialmente si su diagnóstico se retrasa. Aún en este siglo con el desarrollo de técnicas quirúrgicas, modalidades diagnósticas más avanzadas y desarrollo de antibióticos más potentes, la morbilidad y mortalidad relacionadas con lesiones esofágicas continúan siendo altas.

El trauma penetrante del esófago ocurre principalmente en su porción cervical, y la morbilidad está relacionada generalmente a otras lesiones asociadas, principalmente de la vía aérea, lesiones vasculares y de la médula espinal. En varios estudios se ha demostrado que la incidencia de estas lesiones en heridas por arma de fuego transcervicales es de más o menos un $6 \%$, las lesiones debido a trauma contuso son extremadamente raras, se dan en el $1 \%$ de los pacientes que sufren algún tipo de lesión contusa de alta energía ${ }^{1,2,3,4}$.

La perforación en cualquier sitio a lo largo del esófago puede ser una de las lesiones más letales de las que se pueden dar en cualquier otra parte del tubo gastrointestinal y presenta un reto terapéutico para el médico tratante ${ }^{15-17}$. La sintomatología de estas lesiones es variada y poco especifica, por lo que la sospecha clínica es un arma diagnóstica de primer orden, es primordial detectarlas a tiempo ya que con el paso de las horas el daño de los tejidos y la infección de estructuras vecinas puede llevar a la muerte al paciente de forma rápida.

\section{PACIENTES Y MÉTODOS.}

Estudio descriptivo transversal que involucró a los pacientes que ingresaron al servicio de Urgencias Quirúrgicas del Hospital Escuela entre el $1^{\circ}$ de enero de 2008 y el 30 de junio del 2010 con diagnóstico de lesión esofágica.

Se recopiló un total de 11 expedientes de pacientes con lesiones esofágicas de diferente etiología.

Se aplicaron las siguientes variables: nombre, expediente clínico, sexo, edad, mecanismo de lesión, síntomas y signos, hallazgos radiológicos, procedimiento quirúrgico realizado y complicaciones asociadas. La información se recopiló manualmente, utilizando obteniéndola de los expedientes clínicos. Se calculó frecuencias, medianas y porcentajes de las principales variables a través del programa EPI.Info versión 3.5.1 2000.

\section{RESULTADOS.}

De los 11 casos se encontró que el 91\% se presentó en el sexo masculino, que corresponde a 10 pacientes del estudio, la edad media de los pacientes que presentaron estas lesiones fue de 29 años, siendo el menor de 18 años y el de mayor edad de 45 años.

Con respecto a la etiología se evidenció que las heridas por arma de fuego constituyen la principal causa siendo ésta responsable del $64 \%$ de los casos, las lesiones causadas por ingestas accidental de cuerpos extraños representa la segunda causa con un 
$27 \%$ de los casos y en última instancia la ingesta de cáusticos, no se encontró como etiología el factor iatrogénico (por endoscopia) (tabla No1).

\begin{tabular}{lrr}
\hline Etiología de la lesión & \multicolumn{2}{c}{} \\
esofágica & No. Casos & Porcentaje \\
latrogénica & $\mathbf{0}$ & $\mathbf{0 \%}$ \\
cáusticos & 1 & $9 \%$ \\
Cuerpo Extraño & 3 & $27 \%$ \\
HPAB & 0 & $0 \%$ \\
Ruptura Espontánea & 0 & $0 \%$ \\
HPAF & 7 & $64 \%$ \\
Total & 11 & $100 \%$ \\
\hline
\end{tabular}

Tabla No. 1 Etiología de las lesiones esofágicas. Fuente: Expedientes médicos

La porción esofágica más afectada fue la región cervical en un $64 \%$ de los casos solamente el $26 \%$ de los pacientes presentaron lesión en la porción torácica, no se evidenciaron lesiones del esófago abdominal (gráfica No.1).

La sospecha clínica de estas lesiones es importante para su manejo oportuno sin embargo los síntomas y signos de los pacientes tienden a ser vagos y la principal manifestación clínica es el dolor hasta en un $54.5 \%$ de los casos, y el signo más evidente fue el enfisema subcutáneo que se encontró en el $72.7 \%$ de los pacientes.

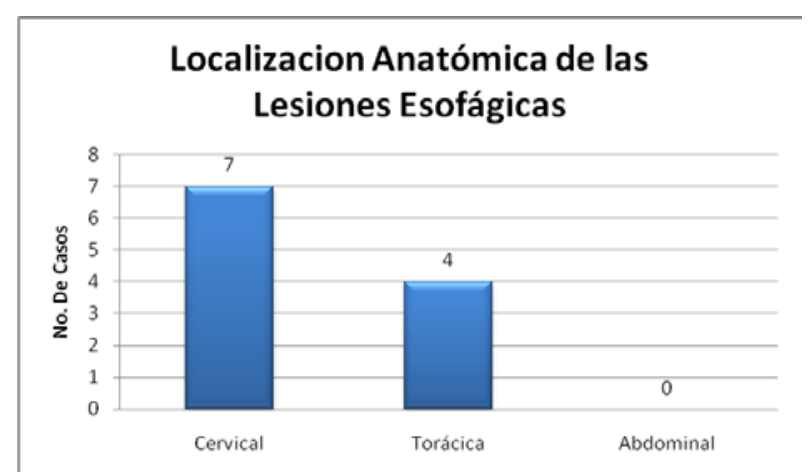

Grafica No.1 Localización anatómica de las lesiones esofágicas
Los métodos diagnósticos más empleados fueron los estudios radiográficos que se realizaron en el $100 \%$ de los pacientes y dentro de los hallazgos más evidentes están el enfisema cervical y el ensanchamiento del espacio retrofaríngeo en un $45.5 \%$ de los casos y en la proyección torácica el enfisema fue el hallazgo más frecuente, sin embargo hasta en un $64 \%$ de los expedientes revisados no se especifican los hallazgos radiológicos. El estudio contrastado más utilizado fue el esófagograma con medio de contraste en el cual se evidenció fuga del medio contrastado en $45.5 \%$ de los pacientes.

El manejo quirúrgico se dio en el $100 \%$ de los pacientes, siendo el procedimiento quirúrgico más empleado el cierre primario de la lesión en el $54.5 \%$, se empleó cierre primario más colocación de un colgajo muscular en el $9.10 \%$ y se decidió realizar exclusión esofágica con gastrostomía para alimentación en el $36.4 \%$ de los pacientes, esto debido al grado de lesión tisular.

Dentro de los hallazgos operatorios se evidenció que las lesiones grado III fueron las más frecuentes en un 50\% habiéndoseles manejado mediante cierre primario, el $25 \%$ con cierre más colocación de colgajo, y sólo un caso en el cual hubo fuga de la rafia se realizó exclusión esofágica, las lesiones grado IV se manejaron en un $100 \%$ con exclusión esofágica (tabla No.2) 


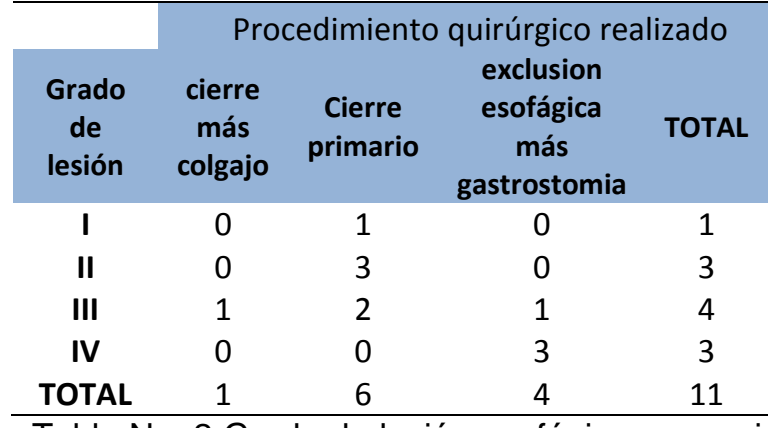

Tabla No. 2 Grado de lesión esofágica y manejo quirúrgico empleado.

Se presentaron complicaciones en 7 casos de las cuales la principal fue la dehiscencia de la rafia y fuga ( $36.4 \%$ ) que se presentó en 4 de los 11 pacientes que debieron ser reintervenidos quirúrgicamente, sin embargo desarrollaron mediastinitis $y$ falla multiorgánica secundaria lo que los llevó a la muerte. Al paciente que presentó lesión por cáusticos se le realizó interposición de colon, posteriormente presentó dehiscencia de la anastomosis por lo que debió ser intervenido nuevamente en este momento se decidió realizar gastrostomía para alimentación, el paciente posteriormente se complicó con neumonía nosocomial que le condujo a la muerte.

De los 11 pacientes manejados el $54.5 \%$ fue dado de alta sin complicaciones médicas y el $45.5 \%$ fallecieron a consecuencia de las complicaciones antes mencionadas (gráfica No.2).

\section{Condición de alta de los pacientes} con lesion esofágica

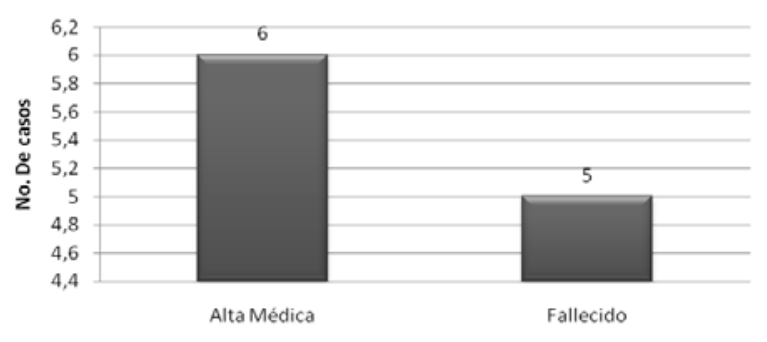

Grafica No. 2 Condición de alta de los pacientes con lesión esofágica.

\section{DISCUSION.}

Las lesiones esofágicas en nuestro medio son poco frecuentes sin embargo su detección tardía puede generar complicaciones letales como la mediastinitis. Un estudio previo realizado en el Hospital Escuela reportó 6 casos de lesiones esofágicas en 22 pacientes que sufrieron heridas por arma de fuego transmediastinales ${ }^{11}$, sin embargo es el único estudio previo realizado en nuestro medio sobre este tema .

De los 11 casos involucrados en el presente estudio la principal causa de las lesiones esofágicas fueron las heridas por arma de fuego, sin embargo aquellas provocadas por cuerpos extraños fueron las que presentaron mayores complicaciones e incluso la muerte de los pacientes ya que en todos estos casos la falta de sospecha y el tiempo de evolución de la lesión hasta su manejo quirúrgico permitió el establecimiento de infecciones severas que conllevaron a una sepsis y falla multiorgánica que concluyó con el fallecimiento de estos pacientes.

La principal sintomatología y signología de estas lesiones fue el dolor y el enfisema subcutáneo, por lo que es necesario llevar a cabo estudios de 
imagen como ser estudios radiológicos simples como una proyección cervical en la cual se puede evidenciar enfisema subcutáneo y aumento del espacio retrofaríngeo sin olvidar que cuando las lesiones son recientes puede encontrarse normal por lo que es necesario apoyarse con estudios contrastados como ser el esófagograma con medio hidrosoluble que en este estudio evidenció fuga del medio en $45.5 \%$ de los casos.

Tal y como se menciona en la literatura internacional ${ }^{1,2,3,4}$ la porción cervical del esófago es la más afectada y sin embargo su abordaje quirúrgico mediante cervicotomía izquierda y la facilidad de reforzar el cierre primario con parches o colgajos musculares es técnicamente más fácil que el manejo de las lesiones de la porción torácica e intraabdominal del esófago.

Las principales complicaciones encontradas fueron la dehiscencia de la rafia y la mediastinitis que conllevó a la muerte a 4 de los pacientes de este estudio. La mortalidad de estas lesiones es elevada por lo que el manejo oportuno es importante para la evolución de estos pacientes.

La principal limitante para la realización del presente estudio fue la recopilación de los expedientes clínicos de los pacientes, ya que en ocasiones se encuentran incompletos, también cabe recalcar la falta de recursos diagnósticos en nuestro medio lo que hace necesario una alta sospecha clínica de estas lesiones para decidir un manejo oportuno.

Es recomendable realizar protocolos de manejo que deben de seguir todos los médicos al estar ante este tipo de lesiones que conlleve a un diagnóstico y a su manejo quirúrgico oportuno con el fin de disminuir las complicaciones letales de esta patología.

\section{CONCLUSIONES.}

Las lesiones esofágicas son entidades raras en nuestro medio sin embargo su baja prevalencia no debe permitir que la baja sospecha de las mismas generen un manejo inadecuado de ellas ya que la mortalidad es elevada. Debe plantearse además la mejor opción quirúrgica en cada caso dependiendo el grado de la lesión, el sitio anatómico y el tiempo de evolución de la misma.

\section{BIBLIOGRAFIA.}

1. James T.et al.: "Esophageal Perforations: New Perspectives and Treatment Paradigms": J Trauma. 2007;63:1173-1184.

2. Italo Braghetto $\mathrm{M}$ et al: "Perforación esofágica. Experiencia clínica y actualización del tema": Rev Méd Chile 2005; 133: 1233-1241

3. Andrade - Alegre R.: " Surgical Treatment of traumatic esophageal perforations. Analysis of 10 cases": CLINICS 2005;60(5):375-80

4. Soto R. Et al: "Lesiones de esófago por trauma externo": Cirujano General Vol. 27 Núm. 1 - 2005

5. Rodrigues A. et al: "Resultados actuales del manejo de la perforación esofágica": Rev. Chilena de Cirugía Vol. 56, Dic 2004. 539-544

6. Pomi R. et al.: " Traumatismos y perforaciones de esófago: diez años de experiencia": Rev Med Uruguay 2005; 21: $308-313$

7. Noriega O. et al: "Perforación Esofágica": Cirugía y Cirujanos 2005: 73: 431 - 435.

8. Nazario A. et al: "PERFORACIÓN ESOFÁGICA TRAUMÁTICA EN UN DECENIO (1990 -1999)": MEDISAN 2002; 6(2):35-41

9. Madiba T. et al: "Penetrating injuries to the cervical oesophagus: is routine 
exploration mandatory?: Ann $R$ Coll Surg Engl 2003; 85: 162-166

10. Christian A Righini et al.: "Cervical cellulitis and mediastinitis following esophageal perforation: A case report": World J Gastroenterol 2008 March 7; 14(9): 1450-1452

11. Neptalí Elvir-Madrid, Francisco AyesValladares, Dagoberto Ordóñez-Rubio: “ LESIONES PENETRANTES DE ESÓFAGO TORÁCICO PRODUCIDAS POR ARMA DE FUEGO. REPORTE DE 6 CASOS" : REV MED POST UNAH Vol. 4 No. 3 Sept- Dic, 1999.

12. Altorjay A, Kiss J, Voros A, Bohak A. Nonoperative management of esophageal perforations. Ann Surg. 1997;225:415-21.

13. Kollmar $O$, Lindermann W, Richter $S$, Steffen I, Pistorius G, Schilling $M K$. Boerhaave's syndrome: primary repair vs. esophageal resection- case reports and meta-analysis of the literature. J Gastrointest Surg. 2003;7:726-34.

14. Wang N, Razzouk AJ, Safavi A, Gan K, Van Arsdell GS, Burton PM. Delayed primary repair of intrathoracic esophageal perforation: is it safe? J Thorac Cardiovasc Surg. 1996;111:11422.

15. Gupta NM, Kaman L. Personal management of 57 consecutive patients with esophageal perforation. Am J Surg. 2004;187:58-63

16. Finley RJ, Pearson FG, Weisel RD, Todd TRJ, Ilves $R$, Cooper J. The management of nonmalignant intrathoracic esophageal perforations. Ann Thorac Surg. 1980;30:575-83.

17. Vasquez J. et al: " Perforación Esofágica y Mediastinitis causada por Espina de Pescado":REV. GASTROENTEROL. PERÚ 2006; 26: 400403

18. Ojima $H$, Kuwano $H$, Sasaki S, Fujisawa $T$, Ishibashi $Y$. Successful late management of spontaneus esophageal rupture using T-tube mediastinoabdominal drainage. Am J Surg. 2001;182:192-6

19. Melero-Sancho LM, Minamoto $H$, Fernández $A$, et al. Descending necrotizing mediastinitis: a retrospective surgical experience. Eur J Cardiothorac Surg 1999;16:200-5
20. Papalia E, Rena O, Oliaro A, et al. Descending necrotizing mediastinitis: surgical management. Eur J Cardiothorac Surg 2001;20:739-42

21. El Oakley RM, Wright JE. Postoperative mediastinitis: classification and management. Ann Thorac Surg 1996;61:1030-6 